\title{
An Unusual Complication of Transthoracic Lung Biopsy
}

Simone Lucia, Daniele Gemma, Chiara Gori, Filippo Rossi Fanelli, Maurizio Muscaritoli

Department of Clinical Medicine, Sapienza - University of Rome, Rome, Italy

\begin{abstract}
:
Objectives: We report a case of a frail older adult affected by Chilaiditi syndrome complicating transthoracic lung biopsy, which has never been presented before in the literature.
\end{abstract}

Materials and methods: After illustration of our case, we review the available literature about Chilaiditi syndrome.

Results: Chilaiditi syndrome is a rare disease characterised by the interposition of colonic segments between the liver and diaphragm associated with mild to severe clinical symptoms.

Conclusion: Although it is uncommon, Chilaiditi syndrome is a clinical condition that should be recognized early and differentiated from other diseases since it may significantly impair a patient's prognosis.

Keywords: Chilaiditi syndrome, colonic interposition, bowel disease, lung biopsy, complication, rare disease

Received: $17 / 12 / 2013$

Accepted: $16 / 04 / 2014$

Published: 09/05/2014

How to cite this article: Lucia S, Gemma D, Gori C, Rossi Fanelli F, Muscaritoli M. An Unusual Complication of Transthoracic Lung Biopsy, EJCRIM 2014;1:doi: 10.12890/2014_000021

Conflicts of Interests: The authors declare that they have no conflicts of interest in this research. 


\section{Case report}

An 84-year-old Caucasian man was admitted to our Department of Clinical Medicine for dyspnoea, which had become progressively severe in the previous 2 days.

He had a past medical history of mild hypertension $(150 / 70 \mathrm{mmHg})$, chronic obstructive pulmonary disease (COPD), polycystic kidney disease associated with chronic kidney disease (CKD) and post-ischaemic dilated cardiomyopathy (ejection fraction, EF=35\%). Five years previously, he had a pacemaker implanted, and when he was an adolescent, he had an appendectomy.

On physical examination, the patient was haemodynamically stable and reported no obvious clinical signs. Complete blood count, liver function tests and urinalysis were normal. Chest $\mathrm{X}$-ray and thorax computed tomography (CT) showed signs of COPD and the presence of two bulky lesions in the apical segments of both the right and left lower lungs (Fig. 1).

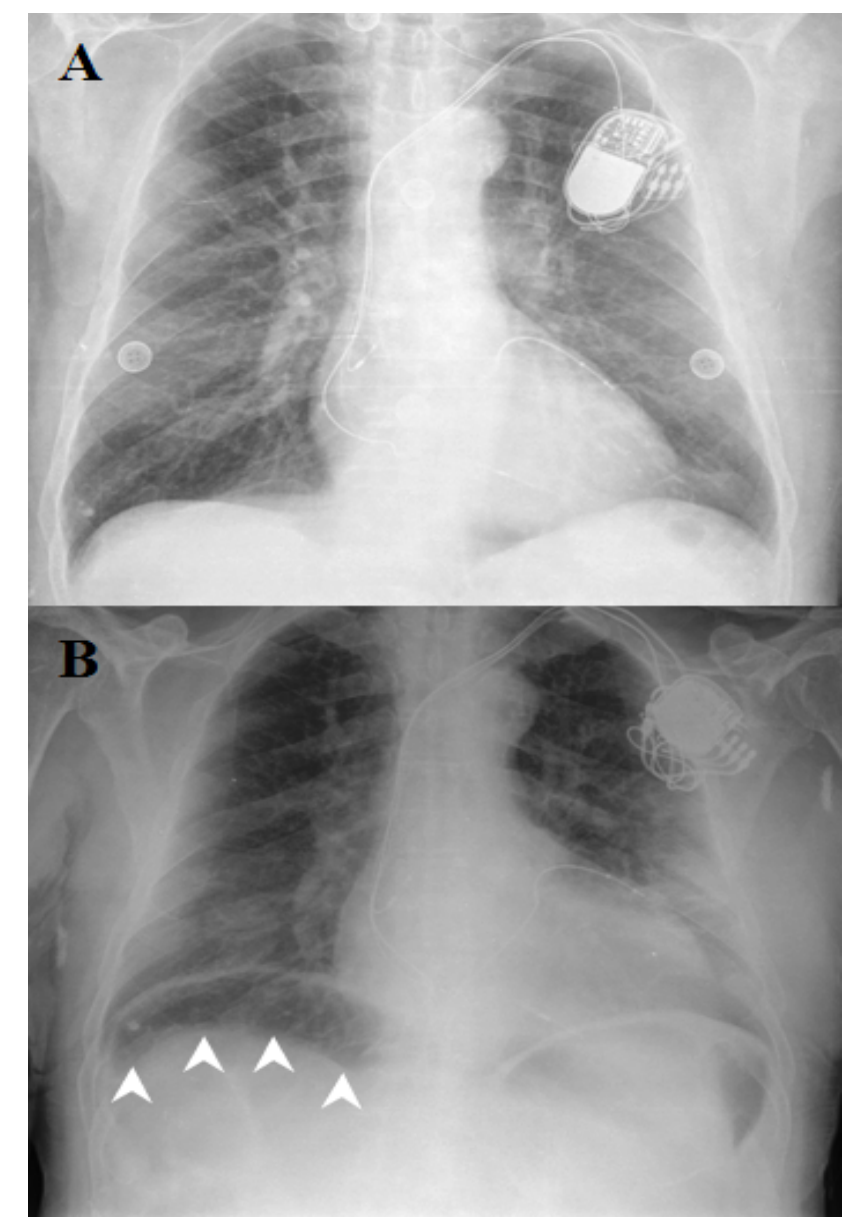

Figure 1: (A) Chest X-ray before lung biopsy.

(B) Chest X-ray after lung biopsy showing the presence of a crescent of air under the right hemidiaphragm as for Chilaiditi sign (arrow).
Lung needle biopsy was performed, confirming the histological nature of the lesions as squamous cell carcinoma. After biopsy, the patient complained of nausea, anorexia and mild, but progressively increasing, non-specific abdominal pain. Chest and abdominal $\mathrm{X}$-ray revealed the presence of air in the right subphrenic region (Fig. 1).

Abdominal CT scan was performed, revealing hepatomegaly and the interposition of a transverse colon segment between the diaphragm and the upper edge of the liver, the so-called Chilaiditi sign (Figs. 2 and 3 ).

As neither clinical symptoms nor Chilaiditi sign were present before the lung needle biopsy, a diagnosis of post-biopsy Chilaiditi syndrome was made. The certainty of the diagnosis was confirmed by comparing the pre- and post-biopsy CT-scan images at the same cross-sectional level (Fig. 2).

The patient was initially treated with 


\section{European Journal \\ of Case Reports in \\ Internal Medicine}

anti-emetic drugs, protonic pump inhibitors and a low-protein diet. Evidence obtained from the literature ${ }^{1}$ suggested the patient should also be managed with bed rest, intravenous fluid therapy and laxatives.

In spite of a slight improvement during the next 5 days, the patient's cardiac function progressively deteriorated, the cardiomyopathy became unresponsive to any inotropic agents, and he died in cardiogenic shock.

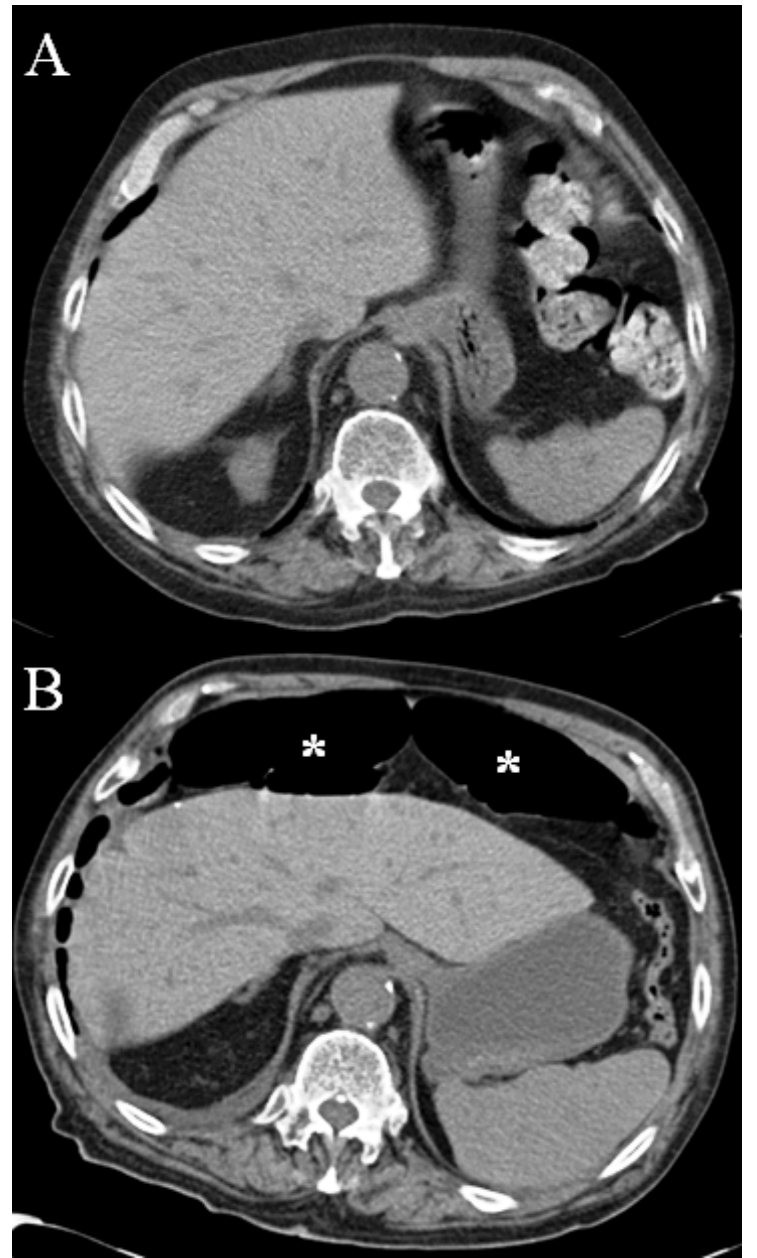

Figure 2: (A) Abdominal CT scan showing the physiological position of the liver as a normal finding before the lung biopsy. (B) Abdominal CT scan made after the lung biopsy showing, at the same axial level, the colonic interposition between the liver and diaphragm as for Chilaiditi sign (asterisks).

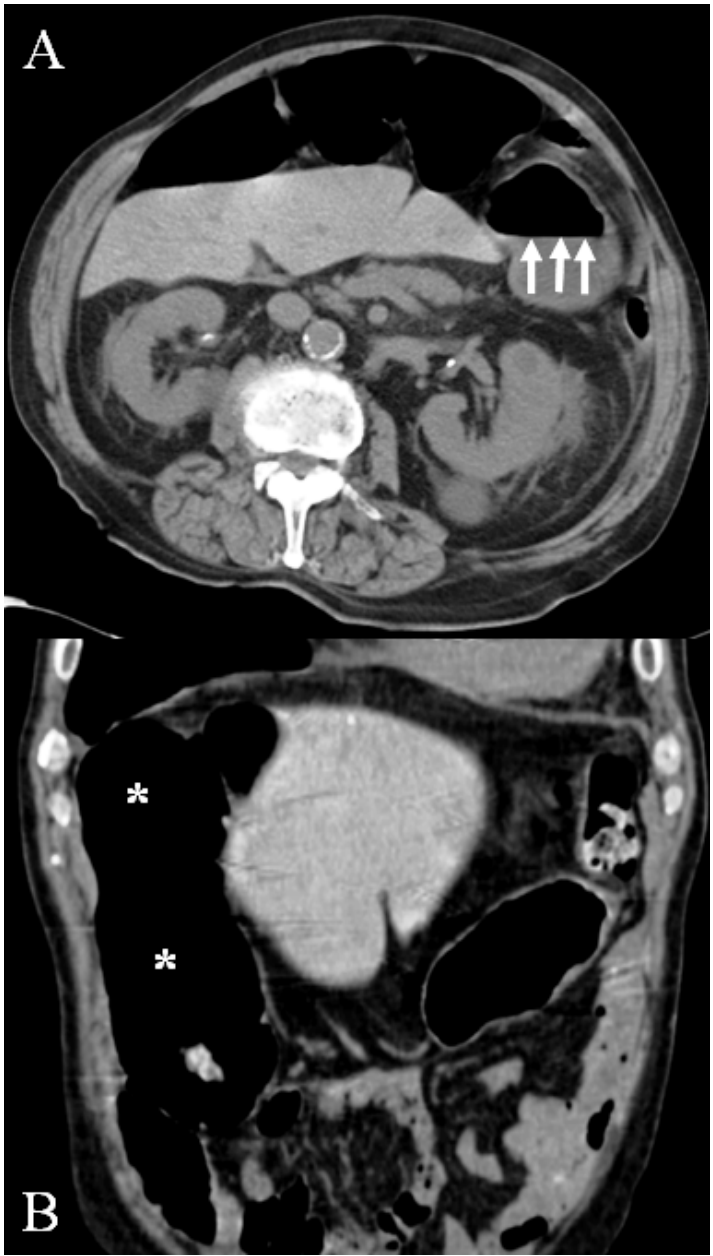

Figure 3: (A) Axial abdominal CT scan showing, in addition to Chilaiditi sign, the presence of an air-fluid level (arrows) as for an intestinal obstruction. (B) Coronal abdominal CT scan showing the interposition of colon between the liver and diaphragm (asterisks).

\section{Review of the literature}

Chilaiditi syndrome is a rare condition that features the interposition of colonic segments between the liver and the diaphragm. It is associated with clinical symptoms like anorexia, nausea, vomiting, 
abdominal pain, abdominal distension, constipation and flatulence. The simple bowel interposition is known as Chilaiditi's sign and it was first described in 1910 by the radiologist Demetrius Chilaiditi ${ }^{1}$.

Since most patients with Chilaiditi syndrome are asymptomatic throughout their lives, clinical symptoms may vary from mild to severe and can occasionally become an emergency demanding an immediate intervention.

The prevalence of Chilaiditi syndrome is difficult to estimate mainly because most of the available data are clinical reports. However, in observational studies the prevalence of hepatodiaphragmatic intestinal interposition ranges from $0.02 \%$ to $3 \%$ in the general population ${ }^{2}$. The bowel segments most commonly found interposed between the liver and diaphragm are the colonic hepatic flexure and the transverse colon, although interposition of the small bowel has also been observed.

Depending on the site of the interposition, Chilaiditi sign may be divided into anterior (more commonly observed) and posterior, while other intestinal interpositions (splenorenal, retrogastric, hepatocaval, retrosplenic and retrorenal) are generally referred to as "non-Chilaiditi" forms ${ }^{3}$.

\section{Pathogenesis}

Although the mechanisms of Chilaiditi syndrome are largely unknown, several predisposing factors, numerous clinical conditions and various medical or surgical procedures have been associated with its pathogenesis.

The predisposing factors may be classified as either congenital or acquired (Table 1).

\begin{tabular}{|l|l|}
\hline \multicolumn{1}{|c|}{ Anatomic predisposing factors } \\
\hline \multicolumn{1}{|c|}{ Congenital variants } & \multicolumn{1}{c|}{ Acquired distortions } \\
\hline Agenesis of the right hepatic lobe & Chronic constipation \\
\hline $\begin{array}{l}\text { Elongation, laxity or absence of the suspensory } \\
\text { ligaments of the transverse colon }\end{array}$ & Severe weight loss \\
\hline Elongation, laxity or absence of the suspensory & Severe COPD \\
\hline ligaments of the falciform ligament & Diaphragm paralysis \\
\hline Dolichocolon & Cirrhosis \\
\hline Congenital elevation of the right hemidiaphragm & Ascites \\
\hline Congenital bowel malposition & Aerophagia \\
\hline & Obesity \\
\cline { 2 - 3 } & Multiple pregnancies \\
\hline
\end{tabular}

Table 1: Predisposing factors of Chilaiditi syndrome 
The anatomic congenital variants are responsible for determining the place of least resistance (i.e. locus minoris resistentiae), predisposing to the pathologic positioning of the colon. On the other hand, the anatomic acquired distortions may be related to clinical conditions responsible for functional disorders (e.g. increased abdominal pressure) and may facilitate the temporary or permanent interposition of the colon between the liver and diaphragm.

Although the causal relation remains unclear, other diseases have been associated with Chilaiditi syndrome. These include cancer (e.g. lung, gastric and colorectal), muscular diseases, scleroderma, congenital hypothyroidism, paralytic ileus, pneumatosis cystoides intestinalis and melanosis coli. In addition, as in our case report, medical and surgical procedures (i.e. lung biopsy, colonoscopy, bariatric surgery, percutaneous transhepatic procedures) may facilitate the occurrence of Chilaiditi syndrome ${ }^{4}$.

Chilaiditi syndrome may be considered as a comorbidity, a complication of medical or surgical procedures, or a negative prognostic factor contributing to deterioration of the patient's clinical picture. Its complications are rare, but may include intestinal obstruction, volvulus, cecal perforation and, more rarely, perforation of the subdiaphragmatic appendicitis. These complications require timely, appropriate and effective interventions.

\section{Diagnosis}

Anamnesis, physical examination and identification of specific imaging signs are the cornerstones of diagnosis of Chilaiditi syndrome.

$\mathrm{X}$-ray identifies the colonic interposition between the liver and diaphragm, mainly showing elevation of the right hemidiaphragm associated with lower liver displacement, bowel distention (pseudopneumoperitoneum) with possible evidence of haustrum and absence of image displacement with changes in the patient's position. However, although abdominal echography and FDG PET have been shown to recognize bowel interposition, abdominal CT scan represents the best imaging method for a differential diagnosis among subphrenic fluid accumulation (e.g. abscesses), pneumoperitoneum and air within the bowel lumen.

Chilaiditi syndrome must be differentiated from bowel obstruction, volvulus, intussusception, ischaemic bowel disease, diaphragmatic hernia and inflammatory conditions (such as appendicitis, diverticulitis, inflammatory bowel diseases).

\section{Treatment}

Little is known about the pathogenesis of Chilaiditi syndrome, and even less is known about how to treat it. It is generally agreed that no treatment is required when Chilaiditi sign is an isolated finding with no or minimal symptoms. In the presence of more serious clinical presentations, the management of Chilaiditi syndrome should be conservative and based on bed rest, nasogastric 
and/or rectal decompression and fluid replacement. Dietary recommendations are higher consumption of fibre and increased fluid intake along with antispasmodic agents 5 . Surgical intervention, such as colon resection, hepatopexy, colopexy and subtotal colectomy, is usually only indicated for the treatment of serious complications and is only considered for severe refractory cases.

\section{Learning Points}

- Chilaiditi syndrome is a rare disease characterised by a colonic interposition between the liver and diaphragm, mainly presenting without evident symptoms, but can have more severe and life-threatening clinical manifestations

- Chilaiditi syndrome may act as a comorbidity of several clinical conditions, as a complication of medical and surgical procedures or even as a negative prognostic factor.

- Chilaiditi syndrome may act as a comorbidity of several clinical conditions, as a complication of medical and surgical procedures or even as a negative prognostic factor.

- Although there are few data on pathogenesis and treatment, awareness of the disease, allowing for a prompt differential diagnosis, may help clinicians in identifying Chilaiditi syndrome and in avoiding emergency complications.

\section{References}

1. Moaven $O$, Hodin RA. Chilaiditi syndrome: a rare entity with important differential diagnoses, Gastroenterol Hepatol (N Y) 2012;8:276-278.

2. Bredolo F, Esposito A, Casiraghi E, Cornalba G, Biondetti P. Intestinal interposition: the prevalence and clinical relevance of non-hepatodiaphragmatic conditions (non-Chilaiditi forms) documented by CT and review of the literature, Radiol Med 2011;116:607-619.

3. Vessal K, Borhanmanesh F. Hepatodiaphragmatic interposition of the intestine (Chilaiditi's syndrome), Clin Radiol 1976;27:113-116.

4. Yin AX, Park GH, Garnett GM, Balfour JF. Chilaiditi syndrome precipitated by colonoscopy: a case report and review of the literature, Hawaii J Med Public Health 2012;71:158-162.

5. Özer C, Zenger S. Chilaiditi syndrome in patient with urological problems: incidental diagnosis on computed tomography, Can Urol Assoc J 2012;6:E75-76. 